\title{
An interpretation of the overionized plasma in supernova remnant $\mathrm{W} 49 \mathrm{~B}$
}

\author{
Xin Zhou ${ }^{1,2,3} \dagger$, Marco Miceli ${ }^{4,5}$ Fabrizio Bocchino $^{5}$, \\ Salvatore Orlando ${ }^{5}$, Yang Chen ${ }^{6,3}, \mathbf{L i ~ J i}^{1}$ and $\mathrm{Ji}_{\mathbf{~ Y a n g}}{ }^{1}$ \\ ${ }^{1}$ Purple Mountain Observatory, Chinese Academy of Sciences Nanjing 210008, China \\ ${ }^{2}$ Key Laboratory of Radio Astronomy, Chinese Academy of Sciences Nanjing 210008, China \\ ${ }^{3}$ Key Laboratory of Modern Astronomy and Astrophysics (Nanjing University), Ministry of \\ Education Nanjing 210093, China \\ ${ }^{4}$ Dipartimento di Fisica, Universit di Palermo Piazza del Parlamento 1, I-90134 Palermo, Italy \\ ${ }^{5}$ INAF-Osservatorio Astronomico di Palermo Piazza del Parlamento 1, I-90134 Palermo, Italy \\ ${ }^{6}$ Department of Astronomy, Nanjing University Nanjing 210093, China
}

\begin{abstract}
W49B is a mixed-morphology supernova remnant (SNR) with the presence of enhanced abundances and overionization confirmed by X-ray observation. For the overionization, a strong radiative recombination continuum (RRC) has been detected and confirmed by $S U Z A K U$ and $X M M-N e w t o n$. Here, we investigate these intriguing observational results through a multidimensional hydrodynamic model that takes into account, for the first time, the mixing of ejecta with the circumstellar and interstellar medium, thermal conduction, and non-equilibrium ionization. The model can reproduce the morphology and the overionization pattern of W49B. We found that the overionized plasma originates from the rapid cooling of the hot plasma originally heated by the shock reflected from the dense ring-like cloud. In addition, based on the most updated ATOMDB (v2.0.2), we calculated the spectrum of one cell in the overionized region from the simulation results at present. We got the overionized spectrum that is in agreement with the observational results. Thus, our primary result indicates that the model is consistent with the observations both spatially and spectrally.
\end{abstract}

Keywords. hydrodynamics; methods: numerical; ISM: individual: W49B(G43.3-0.2); supernova remnant; X-rays: ISM

Recently, strong radiative recombination continua (RRC) were detected by SUZAKU X-ray observations toward W49B (Ozawa et al. 2009) and several other supernova remnants (SNRs), which show the direct signatures of overionization in these SNRs. For W49B, Miceli et al. (2010) further confirmed the RRC feature by a spatially resolved spectral analysis of the XMM-Newton data and found the overionized plasma locate in the center of the remnant and in the western region, but not in the eastern region. The presence of such overionized recombining plasma is a contradictory to the traditional knowledge on SNRs where the plasma should be undergoing an ionizing process until reaching ionization equilibrium, and its origin is not well understood.

W49B is one of mixed-morphology (MM) SNRs (Rho \& Petre 1998) with the presence of enhanced abundances. Its X-ray emission presents a central jet-like feature and a bright limb to the east (Miceli et al. 2006; Keohane et al. 2007). While surrounding the X-ray jet-like structure, a near-infrared barrel-shaped structure with coaxial rings was found (Keohane et al. 2007). It is also found that a strip of shocked molecular hydrogen

$\dagger$ Present address: West Beijing Road 2, Nanjing, China. email address: xinzhou@pmo.ac.cn This work is supported by the 973 Program grant 2009CB824800, NSFC grant 11233001, 100 Talents Program of CAS, and ASI-INAF agreement No. I/009/10/0. 


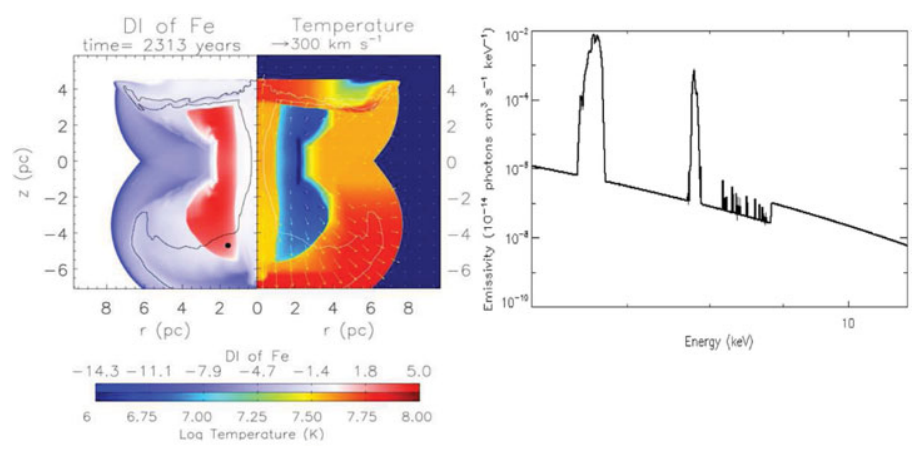

Figure 1. The left panel shows the differential ionization (DI; see Zhou et al. 2011 for the definition) and the temperature distributions in the numerical model, where the black point indicates the position for calculating the spectrum which is shown in the right panel.

is present in the eastern region just outside the shell, implying that the remnant is interacting with a molecular cloud there. These results indicate the remnant is encountering an inhomogeneous environment with particular structures, which are likely to be a remnant of the bipolar wind surrounding the massive progenitor star. In such interaction, the thermal conduction between the shocked molecular material and the inner part of the remnant could be efficient.

In Zhou et al. (2011) we proposed a possible scenario for the origin of overionized plasma in W49B, which is basically consistent with multi-band observational results (Keohane et al. 2007, Ozawa et al. 2009, Miceli et al. 2010). The overionized plasma can be the results of the rapid cooling of the hot plasma originally heated by the shock reflected from a dense ring-like cloud. In particular, we found two different ways for the rapid cooling of plasma to appear: (i) the mixing of relatively cold and dense material evaporated from the ring with the hot shocked plasma and (ii) the rapid adiabatic expansion of the ejecta. Here we show new results on the calculation of X-ray spectra from the numerical model, and we are trying to evaluate this scenario by comparing the numerical model to the observational results spatially and spectrally.

We calculate the X-ray spectrum from a single cell of the numerical model based on the most updated ATOMDB (v2.0.2), which locates in the overionized region with enhanced abundances (see Figure 1). Overionized plasma detected by SUZAKU and XMM-Newton probably locates in this region. The calculated spectrum is consistent with the spectra observed by SUZAKU (see Figure 3 in Ozawa et al. 2009) in many respects, they have corresponding (i) series recombination lines and (ii) prominent RRC feature. We got, for the first time, the model which is spectrally consistent with the observation. These results indicate that our scenario is consistent with the observations both spatially and spectrally. We will further examine the spectral features over the entire remnant in our model for a thorough comparison.

\section{References}

Keohane, J. W., Reach, W. T., Rho, J., \& Jarrett, T. H. 2007, ApJ, 654, 938

Miceli, M., Bocchino, F., Decourchelle, A., Ballet, J., \& Reale, F. 2010, A\&A, 514, L2

Miceli, M., Decourchelle, A., Ballet, J., Bocchino, F., Hughes, J. P., Hwang, U., \& Petre, R. 2006, $A \& A, 453,567$

Rho, J. \& Petre, R. 1998, ApJ, 503, L167

Ozawa, M., Koyama, K., Yamaguchi, H., Masai, K., \& Tamagawa, T. 2009, ApJ, 706, L71

Zhou, X., Miceli, M., Bocchino, F., Orlando, S., \& Chen, Y. 2011, MNRAS, 415, 244 